\title{
Kernos
}

Revue internationale et pluridisciplinaire de religion grecque antique

7| 1994

Varia

Corinne BONNET, Colette JOURDAIN-ANNEQUIN (éds), Héraclès. D'une rive à l'autre de la Méditerranée. Bilan et perspectives

\section{Pierre Brulé}

\section{(2) OpenEdition \\ Journals}

Édition électronique

URL : http://journals.openedition.org/kernos/1117

DOI : 10.4000/kernos. 1117

ISSN : 2034-7871

Éditeur

Centre international d'étude de la religion grecque antique

Édition imprimée

Date de publication : 1 janvier 1994

ISSN : 0776-3824

Référence électronique

Pierre Brulé, «Corinne Bonnet, Colette jouRdain-annequin (éds), Héraclès. D'une rive à l'autre de la

Méditerranée. Bilan et perspectives », Kernos [En ligne], 7| 1994, mis en ligne le 20 avril 2011, consulté

le 21 septembre 2020. URL : http://journals.openedition.org/kernos/1117 ; DOI : https://doi.org/

10.4000/kernos. 1117 
Kernos , 7 (1994), p. 379-416.

\section{REVUE DES LIVRES}

\section{Comptes rendus}

Corinne Bonnet, Colette JouRdaIn-ANnequin (éds), Héraclès. D'une rive à l'autre de la Méditerranée. Bilan et perspectives. Actes de la Table Ronde, Academia Belgica - École française de Rome, 15-16 septembre 1989 à l'occasion du Cinquantenaire de l'Academia Belgica, en Hommage à Franz Cumont, son premier Président, Bruxelles-Rome, 1992. 1 vol. $19 \times 26 \mathrm{~cm}, 361$ p., ill. (Études de philologie, d'archéologie et d'histoire anciennes publiées par l'Institut historique belge de Rome, 28). ISBN : 90-74461-01-8.

C'est à Corinne Bonnet et à Colette Jourdain-Annequin que l'on doit l'heureuse initiative d'avoir rassemblé à Rome en septembre 1989 de très bons connaisseurs de la religion grecque pour évoquer la figure d'Héraclès au travers de ses errances. Le volume d'actes qui en résulte est remarquable, au plan matériel comme au plan scientifique.

Il est superflu d'insister sur l'extraordinaire variété des mythes et des rites où intervient Héraclès; et la dimension géographique, privilégiée ici, accentue cette tendance. Le tableau qui est brossé des voyages héracléens frappe par ses couleurs vives, diverses et contrastées, au point que l'on peut parfois se demander si l'étonnante plasticité du personnage, les formes prises par son culte, par les mythes qui le concernent aux quatre coins du monde connu, ne condamnaient pas d'emblée un tel projet à n'aboutir qu'à la juxtaposition des points de vue. C'est, en outre, généralement la loi du genre. Pourtant, dans ce voyage qui conduit de l'Inde à la mer océane, des lignes de force se dégagent, et c'est ce qui justifie une telle entreprise. La diffusion géographique du personnage, la profusion de ses interprétations, les syncrétismes, tout cela n'empêche pas le lecteur des actes de se surprendre à relever de très nombreux échos entre les analyses et les interprétations de plusieurs participants. On laisse ce volume avec le sentiment d'une cohérence générale qui est une bonne nouvelle s'agissant d'un tel genre.

La proximité des thèmes traités y est évidemment pour beaucoup. Il y a d'abord la place et la fonction d'Héraclès dans le panthéon : F. BADER, Les travaux d'Héraklès et l'idéologie tripartite (7-42), P. LÉVÊQUE, A. Verbanck-PiÉRARD, Héraclès, héros ou dieu ? (43-65). On rattachera à ces mises au point celle de $\mathrm{Cl}$. BAuraIN, Héraclès dans l'épopée homérique (67-109) et de C. BRILlante, La paideia di Eracle (199-222). Le regard porté vers l'Orient, proche ou assez lointain, joue un grand rôle : W. Burkert, Eracle e gli altri eroi culturali del vicino oriente (111-127), 
A. Hermary, Quelques remarques sur les origines proche-orientales de l'iconographie d'Héraclès (129-143), M. Yon, Héraclès à Chypre (145163), C. BONNET, Héraclès en Orient : interprétations et syncrétismes (165-198). Les communications sur les pérégrinations héracléennes en Occident méditerranéen : C. JouRdaIn-ANNEQUIN, Héraclès en Occident (263-291), M. LE GLAy, Héraclès-Hercule en Afrique du Nord (293-308), F. VAN Wonterghem, Il culto di Ercole tra $i$ popoli osco-sabellici (319351) et D. SABBatucCI, Ercole e la fondazione del culto dell'Ara Massima (353-356), offrent au lecteur moins d'occasions d'établir des ponts entre les diverses rives qui sont abordées. C'est sans doute que, de ce côté, la proximité géographique n'est pas autant synonyme de proximité cultuelle. Entre M. Piérart, Les honneurs de Persée et d'Héraclès (23244) et M. Jost, Héraclès en Arcadie (245-261), la parenté ne vient pas que de la proximité géographique, mais d'une dialectique curieuse entre présence et absence du héros en certains lieux sous une forme épichorique ou panhellénique.

Il est bien évident par ailleurs que les thèmes des travaux personnels, alors tout chauds, des responsables du colloque, ceux de C. BONNET sur Melqart (Melqart. Cultes et mythes de l'Héraclès tyrien en Méditerranée, Leuven-Namur, 1988) et ceux de C. JouRDAIN-ANNEQUIN sur l'Héraclès occidental (Héraclès aux portes du soir. Mythe et histoire, Paris-Besançon, 1989) ont contribué à cette unité dont nous nous plaisons à souligner ici l'importance.

$\mathrm{Au}$ travers des Travaux d'Héraclès et des «nombreuses initiations qui leur sont comparables", F. BADER cherche cette fois (après De la préhistoire à l'idéologie tripartite : les Travaux d'Héraclès, in R. BLOCH et alii, D'Héraklès à Poseidon. Mythologie et protohistoire, Genève, 1985, p. 9-124) à montrer comment G. Dumézil a, selon elle, mal évalué l'importance en Grèce de l'idéologie tripartite. Elle porte son attention sur ce qu'il aurait trop délaissé : l'histoire, «l'histoire des économies, sociétés, civilisations... histoire même des conditions d'apparition et de développement de l'idéologie t'ripartite". Mais aussi les mythes "d'acquisition de connaissance". Elle rapproche le cycle des travaux des acquisitions techniques du néolithique, acquisitions elles-mêmes contemporaines de l'expansion indo-européenne : fumure, irrigation (j'entendrais drainage), domestication animale, viennent après la victoire sur les monstres et l'initiation d'Héraclès.

La double nature d'Héraclès fait l'objet d'une double enquête : la part du génétique revient, comme on peut s'y attendre, à P. LéVÊQUE, la part cultuelle à $A$. VerbancK-Pićrard. Du côté du héros au Ile millénaire, pratiquement rien qui ne soit inféré du Ier millénaire, et si l'hypothèse qu'il ait appartenu à la catégorie du «héros [mycénien] cosmique qui purge le monde et repousse les limites de l'oikoumène» nous séduit bien, elle n'est qu'une hypothèse. Et le dieu ? P. LÉvÊQue le situe au sein d'une 
"sainte famille de type néolithique dont les figures féminines, déesse mère / déesse fille, sont représentées par Héra et Athéna». Il conclut qu'on échappe difficilement à l'idée de deux Héraclès mycéniens. L'enquête d'A. VERBANCK-PIÉRARD porte sur un thème où la documentation est fortement contrastée : de l'absence de traces archéologiques aujourd'hui identifiées pour le VIIIe siècle, on passe à la profusion de la fin du Ier millénaire. La réponse de l'A. vient vite : c'est comme

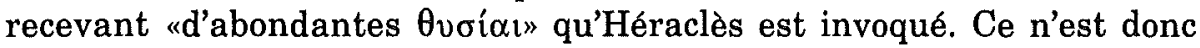
pas de la réalité cultuelle que provient l'ambiguilté. Mais elle est pourtant bien là, dans nos sources. L'A. y voit judicieusement le témoignage de l'existence d'une interrogation sur la curieuse nature du fils de Zeus; interrogation qui, elle-même, alimente la réflexion sur les caractères des personnes divines. Et l'A. évite justement le piège d'un taxinomisme trop étroit en soulignant «les mécanismes d'adaptation interne du polythéisme» et le caractère évolutif des catégories.

C'est au héros seul que s'attache l'étude de C. Brillante sur sa paideia. Comme il conclut, l'analyse révèle qu'elle est conforme au modèle grec de l'éducation héroïque. Avec quelques particularités : entre autres, un projet d'immortalisation qui ne passe pas par l'épreuve du feu et la dation d'un nouveau nom.

Difficile de résumer la mise au point foisonnante de $\mathrm{Cl}$. Baurain sur l'Héraclès homérique. Foisonnante, parce qu'elle est l'occasion pour l'A. de s'engager dans des débats annexes, mais inévitables, où il retrouve des problématiques toujours d'actualité, après Coldstream et Snodgrass, avec les recherches de Th. Hadzisteliou PrICE (ainsi dans G. Bower-Sock et alii (éd.), Arktouros; Hellenic Studies pres. to B. Knox on the Occasion of his 65th Birthday, Berlin-New York, 1979, p. 219-228), de J. Withley (JHS, 1988, p. 173-182) et de I. MorRIS (Antiquity, 62, 1988, p. 750-761).

Héraclès et le Proche-Orient, vaste programme, sujet des communications de W. Burkert et de A. Hermary. Pour le premier, il s'agit de poursuivre la mise en parallèle de la geste d'Héraclès avec celles que donnent à lire des mythes mésopotamiens, particulièrement ceux de «Gilgamesh le héros et de Ninurta le dieu». Cette communication illustre un aspect de l'enquête plus générale que conduit l'A. sur les parentés entre les imaginaires archaïques grecs et ceux procheorientaux du IIe millénaire (Oriental and Greek Mythology: The Meeting of Parallels, in J. BREMMER (éd.), Interpretation of Greek Mythology, Beckenham, 1987, p. 10-40). Il y retrouve des structures proches et des éléments identiques. Violence et victoire marquent les interventions de Gilgamesh et Ninurta. Il note le caractère, particulier à la figure d'Héraclès, du «non-hoplites, [de] l'anti-hoplites". Du côté de l'iconographie, on est depuis longtemps à l'affût d'éventuelles influences proche-orientales. Déjà, nous dit A. Hermary, Raoul- 
Rochette avait mis en évidence la filiation entre des tueurs de lion sur des coupes d'argent chypro-phéniciennes et l'Héraclès de l'époque archaïque. L'A. examine ensuite l'iconographie chypro-phénicienne de la "journée de chasse" (premier quart du VIIe s. ?) pour laquelle il conclut prudemment à une scène héracléenne traduite dans le langage artistique d'artisans phéniciens. Dans ces échanges entre culture proche-orientale et hellénisme, Chypre tient évidemment une place de choix. M. Yon montre que l'iconographie y offre dès l'époque archaïque des types susceptibles d'être mis en rapport avec Héraclès (barbu, avec, puis sans arc). À partir de 500 se répand (surtout dans les régions de culture phénicienne) le motif du «jeune homme au lion», «il correspond en fait à un Melqart».

La communication de C. BONNET est une mise au point, un état de la question. Qu'est-il résulté des diverses rencontres de l'Héraclès grec, transporté par les conquérants macédoniens jusqu'en Asie centrale, avec les croyances et les divinités locales? Cela nous vaut un grand et beau voyage où les principaux acteurs sont la propagande royale, l'extraordinaire plasticité d'Héraclès et les divinités diverses rencontrées sur le chemin. Cela va du Melqart tyrien, au Nergal de Palmyre et d'ailleurs, au Verethragna, le correspondant parthe du héros en Mésopotamie, en Commagène et en Iran, et, lorsqu'on atteint les plaines de l'Inde, à Krishna. Ses "succès" sont surtout ceux des armées d'Alexandre pour lesquelles la figure héroïque valait surtout par sa force belliqueuse destructrice. C'est par elles qu'il parvient à vaincre les résistances. Mais lorsque l'on considère le bilan, le tableau est tout à fait curieux. Ses interprétations, syncrétismes et emprunts d'Héraclès lui font faire le grand écart : ici héros souffrant, ailleurs "prophylactique et apotropaïque», là "force vitale redoutable».

Revenons en Grèce, plus spécialement dans le Péloponnèse. On sait l'importance prise par la notion de parenté dans les relations entre les cités de l'antiquité, surtout aux époques hellénistique et romaine. Dans cette ambiance culturelle, établiŕ la «bonne naissance» d'un individu, d'une famille, d'une cité, c'est les ranger parmi les élites, celles qui, en outre, se voient gratifiées d'honneurs héroïques. À Argos, ce sont les "honneurs de Persée et d'Héraclès». L'expression est curieuse parce que si Persée, comme héros argien, y tient une place naturelle, "Héraclès est personnellement absent des récits que les Argiens ont attachés à leurs monuments" (c'est-à-dire à leur passé tel que le livre Pausanias). Le fait est indubitable, mais curieux : comment expliquer le rejet à Argos de ce qu'on pourrait appeler ailleurs dans le Péloponnèse "l'explication par Héraclès" ? La question que pose M. Pí́rart est difficile. Plus généralement, pourquoi les cartes des exploits d'Héraclès et celle de ses cultes ne coïncident-elles pas ? C'est sans doute du côté de l'histoire que se trouve la solution. M. Jost retrouve le thème en 
Arcadie : à Tégée, Augé est prêtresse d'Athéna Aléa, elle est liée à un culte d'Ilithye, mais Héraclès qui la viole «fait un peu figure d'étranger de passage". D'autres mythes ont une "diffusion panhellénique", mais "pas d'audience locale".

Symétrique du voyage de C. Bonnet, celui auquel C. JourdarnANNEQUIN nous invite vers "Gadès pour les troupeaux de Géryon, [vers] Lixos pour les Hespérides". Symétrique aussi, dans cette direction, le recours à l'histoire, qui ne va pas sans poser des problèmes méthodologiques. L'A. affiche avec netteté son opinion : «si... le voyage vers l'Ouest d'Héraclès ne peut, en aucun cas, être une création coloniale, ses variantes, en revanche, le seraient bel et bien". Cet Ouest si lointain du mythe apparaît comme une marge, mais luxuriante, aux richesses infinies. Là Héraclès - celui de Diodore surtout - qui purifie, pacifie, rend les terres fertiles, défend contre le danger représenté par "l'indigène inhospitalier et xénophobe», bref celui qui colonise l'archégète -, rencontre Melqart auquel il s'unit. Mais c'est en même temps en ces lieux que se termine le mythe. Il s'y termine en leur prêtant les caractères d'un au-delà.

En Afrique du Nord, le mélange des personnalités rend difficile la distinction entre Melqart, aux traits trop proches d'un Héraclès grécoitaliote, et Héraclès lui-même. La plasticité fonctionnelle est là aussi remarquable. Il y a l'Héraclès «ingénieur des Ponts", perceur de routes comme ailleurs en Occident, le dieu fondateur de villes et donc des élites qui les dirigent (à Leptis Magna, il est dit «Seigneur» de la ville), le spécialiste des eaux, des thermes : au IIIe siècle, voilà que l'on rencontre un Hercule mystique, chthonien; l'ambiance éleusinienne, le rapprochement avec Bacchus témoignent de l'entrée du héros dans une religion de salut.

L'actualité héracléenne aujourd'hui, c'est la tenue d'un deuxième colloque Héraclès à Grenoble en octobre 1992 organisé par C. Bonnet et C. Jourdain-Annequin : Héraclès et le féminin. Ses actes sont sous presse dans la même collection, Nous les attendons impatiemment.

Pierre BRULÉ

(Université de Rennes 2)

Fritz Graf (éd.), Klassische Antike und neue Wege der Kulturwissenschaften. Symposium Karl Meuli (Basel, 11.-13. September 1991), Basel, Schweizerische Gesellschaft für Volkskunde, 1992, 1 vol. 15,5 x $22,5 \mathrm{~cm}, 221$ p. (Beiträge zur Volkskunde, 11). ISBN : 3-908121-46-5.

Karl Meuli, né le 15 septembre 1891, mourut le 1er mai 1968. Pour le centième anniversaire de sa naissance a été organisé à Bâle, où il avait été 37 ans professeur de gymnase et où il avait eu une brillante carrière 